\title{
Exploring Mobile Game Interactions
}

\author{
Pitsanu Chaichitwanidchakol, Witcha Feungchan \\ Departement of Computer Engineering, Faculty of Engineering, Khon Kaen University, Thailand
}

\begin{tabular}{l} 
Article Info \\
\hline Article history: \\
Received Apr 4, 2018 \\
Revised Jun 17, 2018 \\
Accepted Jul 5, 2018 \\
\hline
\end{tabular}

Keyword:

Game interactions

Interaction design

Interaction techniques

Mobile games

\begin{abstract}
The mobile game industry has been growing rapidly in both the number of games and revenues. Choosing the right interactions for a game has become a major challenge for developers. Some developers use inappropriate interactions in their games which causes them to be less fun than they should be. This research focuses on gathering and defining possible mobile game interactions so as to guide and enable designers and developers to choose the right interactions for their games. The researchers have extensively reviewed and explored various mobile game interactions both through research studies and through existing mobile games. Subsequent to observations, mobile game interactions were then categorized as follows: 1) Touch interaction 2) Motion/Movement interaction 3) Video interaction 4) Sound interaction 5) Special purpose interaction 6) Location interaction 7) Electroencephalography (EEG) interaction 8) Date/Time interaction 9) Weather interaction 10) Light interaction 11) Proximity interaction 12) Network interaction 13) Social interaction and 14) Bioinformatics interaction. These 14 interactions can be used to support gameplay, ideas, and innovation of mobile games.
\end{abstract}

Copyright $\odot 2018$ Institute of Advanced Engineering and Science. All rights reserved.

\section{Corresponding Author:}

Pitsanu Chaichitwanidchakol,

Departement of Computer Engineering,

Khon Kaen University,

123 Moo 16 Mittapap Rd., Nai-Muang, Muang District, Khon Kaen 40002, Thailand.

Email: pitsanu.c@kkumail.com

\section{INTRODUCTION}

Games are for playing, and bringing joy and entertainment to players [1]. Games have grown to become a major electronic entertainment business that engages a wide range of players around the world due to its availability through various platforms. The most widely used platform is the mobile platform and there is a tendency for the number of mobile users to increase every day [2]. Games on this platform are called mobile games. Mobile games include video games that are played on smartphones, feature phones, smartwatches, PDAs, tablets, or handheld devices. Currently, a huge amount of revenue is generated from mobile games and advertising in mobile games [3]. This study solely focuses on mobile games since most smartphones are equipped with special features such as a touch screen and various sensors (accelerometer, gyroscope, magnetometer, etc.) which can give rich digital interactivity with the surrounding environment, daily activities, social interactions, and so on [4]. In addition, wearable devices like heart rate monitoring devices and step counter devices can also bring new experiences to smartphone game players. With these reasons, most game developers now prefer to develop games on smartphones. Tetris, the first mobile game on Hagenuk MT-2000 was introduced in 1994. Later in 1997, Nokia presented Snake [5], a mobile game on Nokia 6610, and had made great success at that time as a result from the 350 million devices sold. From then on, many more mobile games were developed using Java language. At that time, the only interaction between players and the the mobile games on those devices was just through button pressing. In 2009, Apple introduced the iPhone, a smart phone with a touch screen and special sensors such as an accelerometer, 
gyroscope, and GPS. This led to many new interactions between the user and the mobile games. Current smartphones are equipped with even more various functions and new interactions. These features in mobile games give players a new, challenging experience as well as increase their enjoyment.

In [6], mobile game interactions were divided into three types: 1) touch gesture 2) accelerometer and 3) simulated button, while in [7], mobile game interactions were divided into three different types: 1) key touch 2) motion and 3 ) gesture (touch). The categorizations of these two studies were very similar. However, [8], which studied game interactions in various platforms, had a different aspect of categorization for game interactions. The study of [8] used controlling devices as the criteria, dividing game interactions into five types: 1) emulated devices 2) movement tracking 3) pen-based touch screens 4) voice analysis and 5) multimodal tabletop interfaces. In [9], two types of interactions were defined for a Chinese cooking game which included: 1) touch gesture and 2) motion gesture. [10] defined interactions for an augmented reality game by using a video-based tracking method. [11] used gestures with serious games on mobile devices and had defined interaction systems of people and gesture interfaces into three types: 1) visual recognition 2) handheld devices and 3) hybrid (data glove and visual recognition). Finally, [12] had presented eight interactions including: 1) multi-touch, 2) handwriting recognition, 3) eye gaze tracking, 4) brain-computer interface, 5) inertial sensor, 6) magnetic sensor, 7) speech, and 8) data glove.

This study had aimed to gather and define all possible interactions for mobile games by studying related existing researches along with new mobile technologies to reveal some undiscovered interaction techniques. The study also introduced a list of interactions for mobile games for designers and developers to use as a guideline to develop more novel and interesting games by choosing the appropriate interactions, depending on their design concept and the type of game.

As a result, the study had exclusively presented 14 interaction techniques for mobile games. Seven interactions were defined from related past researches including: 1) Touch interaction 2) Motion/Movement interaction 3) Video interaction 4) Sound interaction 5) Special purpose interaction 6) Location interaction and 7) Electroencephalography (EEG) interaction. Apart from the techniques mentioned, the researchers of this study had discovered seven additional interactions which included: 1) Date/Time interaction 2) Weather interaction 3) Light interaction 4) Proximity interaction 5) Network interaction 6) Social interaction and 7) Bioinformatics interaction. With these 14 techniques, game designers and developers can choose the appropriate interactions to apply which will not only help reduce the design and development time, but also provide new game experiences to the players.

\section{IDENTIFICATION OF MOBILE GAME INTERACTIONS}

Game interaction refers to how the player interacts with the game in some manner such as pressing the button, tilting the device, or speaking. Game interactions can be divided into two major types [13]:

a. Non-Natural interactions which are interactions that mainly uses hands or fingers. Players can interact with the game though interactive devices such as a mouse, keyboard, or joystick.

b. Natural interactions which are techniques that mimic real-world interactions via body movements and actions that are similar to those used for the same task in the physical world. It can be achieved by either holding or not holding a controlling device. If there is no controlling device, there would be sensors like depth sensors or cameras, installed to detect player' movements. If a controlling device is required, the device would be equipped with some sensors (for example, accelerometer, gyroscope, proximity sensor, magnetometer, ambient light sensor, thermometer, or bioinformatics sensors). The pattern of natural interactions include body movements, head movements, postures, gestures, and sounds.

Natural and non-natural game interactions both occur on mobile games. This is due to the small size of a smartphone, requiring players to hold the device continuously while interacting with the game. Smartphones are also equipped with sensors that enable them to use natural interactions conveniently. For example, in a racing game, an accelerometer is used to detect tilting in the device in order to turn the steering wheel for controlling the car. Moreover, most modern smartphones have no physical buttons since the touch screen with an onscreen keyboard is used as the main input. If a user needs to use physical buttons, a game pad or a joy stick can be connected to the device via Bluetooth or data port.

In Figure 1, the interaction of mobile games is divided into two parts, the player and the mobile device. There are three types of interactions between the player and the mobile device. Firstly, visual interaction provides the main interaction by giving visual feedback to the player via the device's screen. Secondly, auditory interaction normally utilizes the microphone and the speaker for the player to interact with the game. Lastly, haptic interaction mainly uses touch to interact via finger, hand or skin with vibration feedback. 


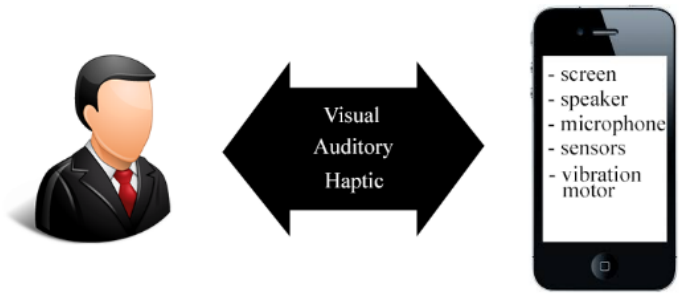

Figure 1. Mobile game interactions between player and mobile device

Based on relevant research studies, it is possible to summarize the interaction techniques for mobile games as shown in Table 1.

Table 1. Interactions from related Researches

\begin{tabular}{cl}
\hline Author & \multicolumn{1}{c}{ Interactions } \\
\hline$[6]$ & 1) touch gesture, 2) accelerometer and 3) simulated button \\
{$[7]$} & 1) key touch, 2) motion and 3) gesture (touch) \\
{$[8]$} & 1) emulated devices, 2) movement tracking, 3) pen-based touch screens, \\
& 4) voice analysis and 5) multi-modal tabletop interfaces \\
{$[9]$} & 1) touch gesture and 2) motion gesture \\
{$[10]$} & 1) video-based tracking \\
{$[11]$} & 1) visual recognition, 2) hand-held devices and 3) hybrid \\
{$[12]$} & 1) multi-touch, 2) handwriting recognition, 3) eye gaze tracking, \\
& 4) brain-computer interface, 5) inertial sensor, 6) magnetic sensor, \\
\hline
\end{tabular}

However, categorizations of game interactions from previous studies were still unclear and incomplete and were not described in much detail. The researchers of this study had therefore gathered and introduced new types of game interaction for mobiles, resulting to 14 different types of interactions as shown in Table 2 and described as follows:

Table 2. The comparison between the Proposed Interactions and Previously Related Researches

\begin{tabular}{lcccccccc}
\hline \multicolumn{1}{c}{ Interactions } & {$[6]$} & {$[7]$} & {$[8]$} & {$[9]$} & {$[10]$} & {$[11]$} & {$[12]$} & Proposed \\
\hline 1. Touch & $\checkmark$ & $\checkmark$ & $\checkmark$ & $\checkmark$ & - & - & $\checkmark$ & $\checkmark$ \\
2. Motion/Movement & $\checkmark$ & $\checkmark$ & $\checkmark$ & $\checkmark$ & - & $\checkmark$ & $\checkmark$ & $\checkmark$ \\
3. Video & - & - & - & - & $\checkmark$ & $\checkmark$ & $\checkmark$ & $\checkmark$ \\
4. Sound & - & - & $\checkmark$ & - & - & - & $\checkmark$ & $\checkmark$ \\
5. Special purpose & - & - & $\checkmark$ & - & - & $\checkmark$ & $\checkmark$ & $\checkmark$ \\
6. Location & - & - & - & - & - & - & $\checkmark$ & $\checkmark$ \\
7. EEG & - & - & - & - & - & - & $\checkmark$ & $\checkmark$ \\
8. Date/Time & - & - & - & - & - & - & - & $\checkmark$ \\
9. Weather & - & - & - & - & - & - & - & $\checkmark$ \\
10. Light & - & - & - & - & - & - & - & $\checkmark$ \\
11. Proximity & - & - & - & - & - & - & - & $\checkmark$ \\
12. Network & - & - & - & - & - & - & - & $\checkmark$ \\
13. Social & - & - & - & - & - & - & - & $\checkmark$ \\
14. Bioinformatics & - & - & - & - & - & - & - & $\checkmark$ \\
\hline
\end{tabular}

\subsection{Touch interaction}

Touch interaction is the interaction that uses fingers to touch the touchscreen to interact with the game. There are many techniques that uses in this type of interaction such as multi-touch, which uses multiple fingers to touch the screen at the same time to interact and touch gesture recognition, which uses more than one finger to touch the screen and drag in specified patterns to invoke some actions. An example is the zoom gesture which uses two fingers to touch the screen and then drag away from one another to zoom. Pre-touch sensing [14] detects fingers that are close to the screen without the need to physically touch it as shown in Figure 2. 3D touch [15] uses a screen that can measure the pressure on the screen and display different interactions depending on the haptic feedback [16], [17]. This technique of screen interaction makes 
the user feel that the touch physically occurred (like when pressing the physical button). Lastly, rich touch [18] is the technique to sort the object by using fingers, nails or knuckles to touch the screen.

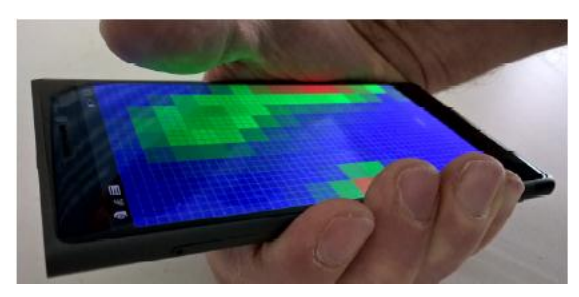

Figure 2. Touch interaction (Pre-touch [14])

\subsection{Motion/movement interaction}

Motion/Movement interaction is the interaction that uses movements or motions of the mobile device to interact with the game. For this kind of interaction, the device must be equipped with motion detection sensors such as an accelerometer, gyroscope, magnetometer, or camera. Examples of techniques using this interaction are motion detection [19] which senses the movement of the device; tilting [20], [21] as shown in Figure 3, which senses declination of the device; gesture recognition [21], [22] which compares capability of sensors to define the interaction patterns; GripSense [23] which uses sensors and touchscreen to detect hand gestures and pressure on the device; MagicPhone [24] which uses accelerometer and magnetometer to indicate the directions and locations that the mobile device is pointing to to control other electrical devices; camera motion [25] which uses a camera to detect movements by using two photos to calculate the directions of movement; and Leap Motion [26] which is a device connected with the mobile device via Bluetooth for detecting hand gestures and movements.

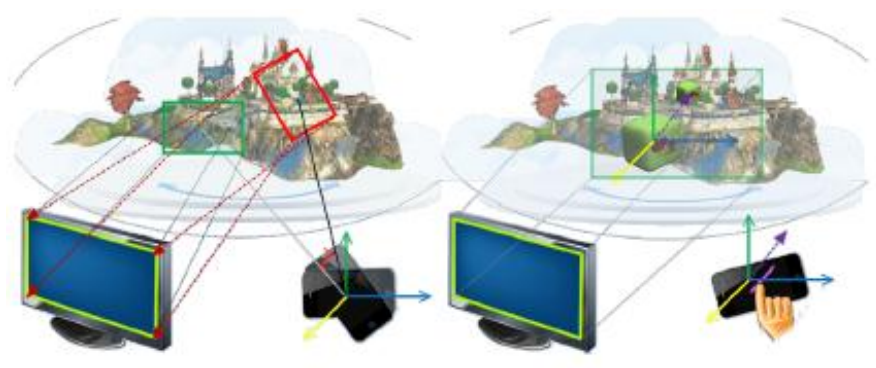

Figure 3. Motion/movement interaction [20]

\subsection{Video interaction}

Video interaction is the interaction that uses a video camera to detect facial expressions [27] or to insert a virtual image (2D or 3D) to the real world (called Augmented Reality (AR) and Virtual Reality $(\mathrm{VR})$ ). AR and VR enables the user to see the virtual image overlapping with the real world scene when looking through the camera. Pokemon Go! [28] is a good example for video interaction as shown in Figure 4.

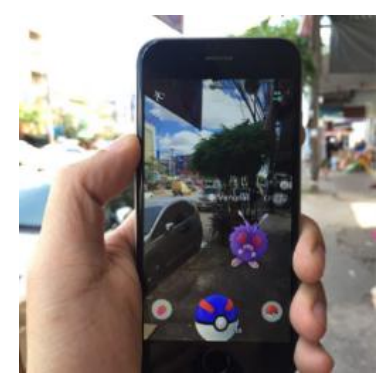

Figure 4. Video interaction (Pokémon GO [28]) 


\subsection{Sound interaction}

Sound interaction is the interaction that uses the player's voice to interact with the game. The gadget required for this interaction is a microphone. The concept of this interaction is to use voices from the player to process the following techniques; voice recognition [29] or speech recognition [30] which detects what the player says and then interact with the game depending on what the speech is (for example, the player says jump to make the game character jump); sound processing [31] which processes the sounds from the player; vocal processing (Karaoke fame) which processes singing voice and then grades its quality from the pitch key or amplitude; and vocal emotion [32] which detects emotions from the player's speech to interact with the game as shown in Figure 5.

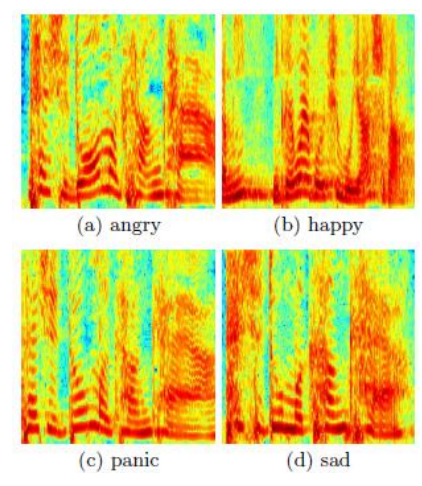

Figure 5. Sound interaction (vocal emotion [32])

\subsection{Special purpose interaction}

Special purpose interaction is the interaction that uses a special device connected to the mobile device mostly through an AUX channel or Bluetooth to transmit electronic signals to the mobile device for processing. These special connecting devices are created to imitate special purpose devices such as a joystick, guitar, or smart watch. The connecting devices enhance the players experience and make them feel, for example, like they were really playing a guitar. Card swipe devices also uses a special equipment to read data from a card and transmit it to the mobile device via AUX channel. Alcohol detection equipment uses a special device to measure the alcohol level from the breath and transmit the data to the mobile device via AUX channel or Bluetooth channel. Pokémon GO Plus [33] (shown in Figure 6) is used to notify about an event occurring in the Pokémon GO.

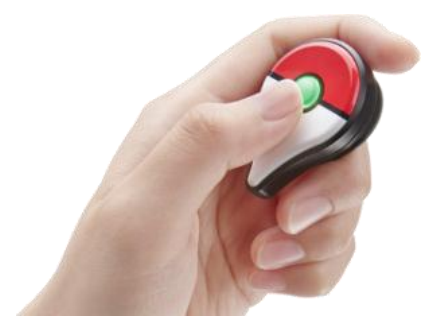

Figure 6. Special purpose interaction (Pokémon GO Plus [33])

\subsection{Location interaction}

Location interaction is the interaction that uses the current location of the player to interact with the game. This kind of game is called a location-based game such as Moomin Move [34] as shown in Figure 7. Techniques used in this interaction are GPS tracking, which uses GPS to identify geo-location and menetometer to identify direction, iBeacon tracking, which uses iBeacon to communicate with the mobile device and calculate the distance between the device and iBeacon, Network-based tracking, which identifies geo-location using the IP (Internet Protocol) address of the network, SIM-based tracking, which identifies geo-location using three phone antennas instead of the satellite, and Altitude measurement, which measures the altitudes to interact. 


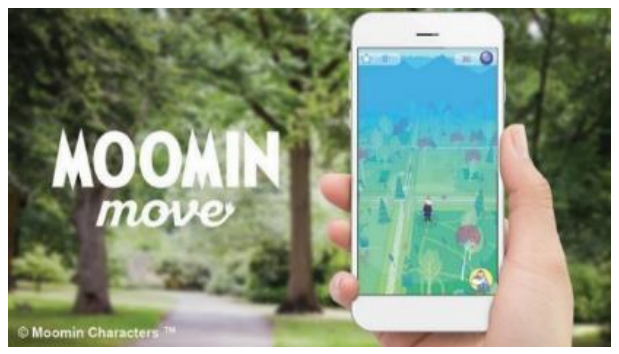

Figure 7. Location interaction (Moomin Move [34])

\subsection{Electroencephalography (EEG) interaction}

EEG is the interaction using brain waves to interact with the game. To interact, the player must wear a gadget to detect the brain waves and send them to the mobile device for controlling the game. EEG control technique [35] is used to detect the patterns of the brain wave and match it with defined patterns to invoke actions such as turning left or right (shown in Figure 8).

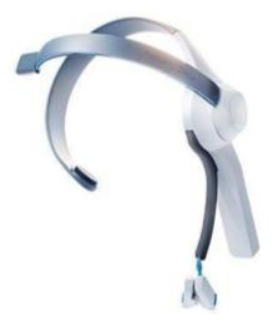

Figure 8. EEG interaction (NeuroSky [35])

\subsection{Date/time interaction}

Date/Time interaction is the interaction that uses date and time to interact with the game such as using time to define a day or night scene in accordance with the real time. By using this type of interaction, seasons and festivals can be defined in the game depending on current real-world dates such as the Christmas event in RO: Idle Poring [36] as shown in Figure 9.

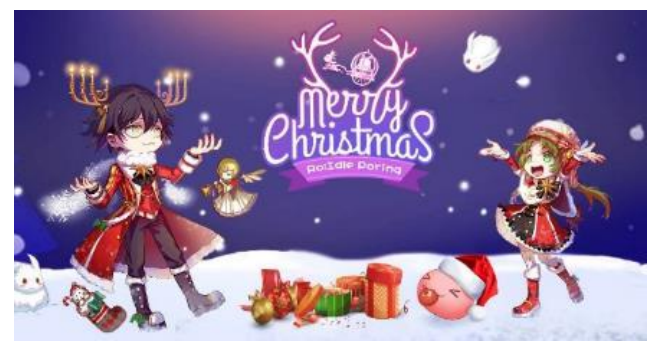

Figure 9. Date/time interaction (RO: Idle Poring [36])

\subsection{Weather interaction}

Weather interaction is the interaction that uses real-time weather to interact. The mobile device can measure the weather with sensors such as the temperature sensor, humidity sensor and barometer. Examples of this kind of interaction are game weather control, temperature measurement, humidity measurement and season changes on game scenes such as the Weather boost system in Pokémon GO [28] as shown in Figure 10. 


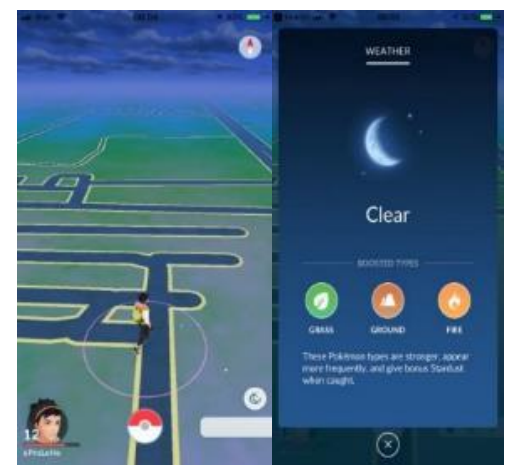

Figure 10. Weather interaction (Pokémon GO [28])

\subsection{Light interaction}

Light interaction is the interaction that detects the light from the actual environment to interact with the game. Ambient light measurement is used to adjust the game scene brightness and Day/Night scenes depending on the light in the actual environment. For example, Boktai (Figure 11) on Game Boy Advance uses a light sensor to measure the amount of sunlight for changing the in-game weapon.

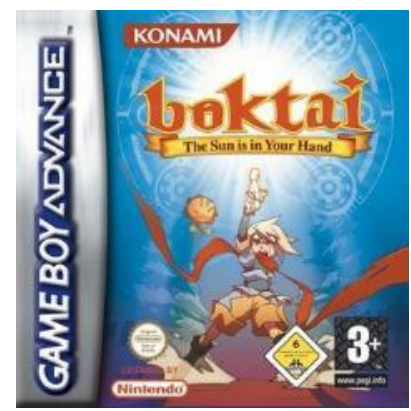

Figure 11. Light interaction (Boktai: The Sun Is in Your Hand [37])

\subsection{Proximity interaction}

Proximity interaction is the interaction that detects the length or distance to interact with the game. Techniques used in this interaction include proximity measurement, which measures the distance between an object and the proximity sensor to interact, Beacon tracking, which is the communication technique used in Beacon devices by broadcasting to other mobile devices in range and enabling them to receive data, NFC tracking, which uses NFC (Near Field Communication) for transmitting in short range with high security such as making payment (shown in Figure 12) or authentication, and hall sensor tracking, which detects the open/close status of the mobile case cover.

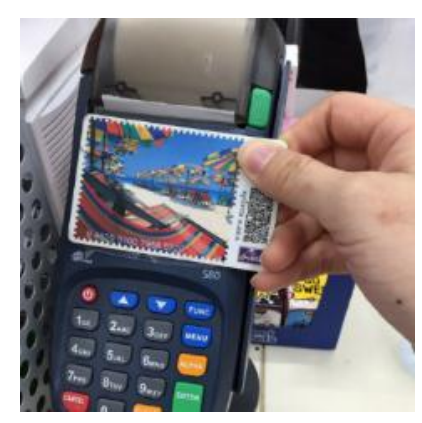

Figure 12. Proximity interaction (NFC) 


\subsection{Network interaction}

Network interaction is the interaction that uses network to interact. The concept is to detect the network usage. If the player is in the network, the player will receive a special privilege or message that players in other networks cannot receive. Examples of techniques used in this interaction are IP Bonus, which uses IP to separate the user group (if the players' IPs are in the same group, the defined interaction will occur), and Beacon broadcast message, which uses beacon to send a message to the users in the communicable range of beacon. The example of Beacon is shown in Figure 13.

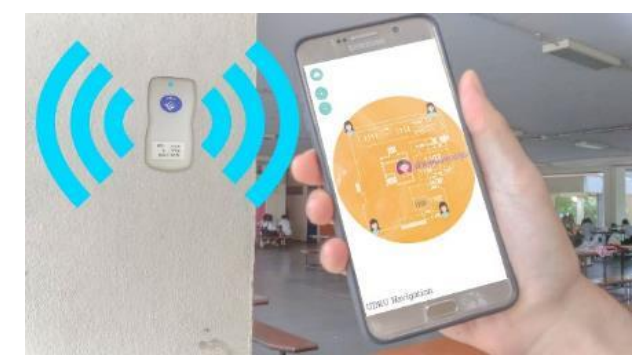

Figure 13. Network interaction (iBeacon [38])

\subsection{Social interaction}

Social interaction is the interaction between players in the game which can be divided into two modes: competitive and cooperative. Social interaction will allow players to have more fun as the game is played by several people who can either help each other to complete the mission or to compete for the race. This type of gameplay will give players a more exciting experience than playing alone. A good example of games using this technique is Alphalt Adrenaline 6 [39] as shown in Figure 14.

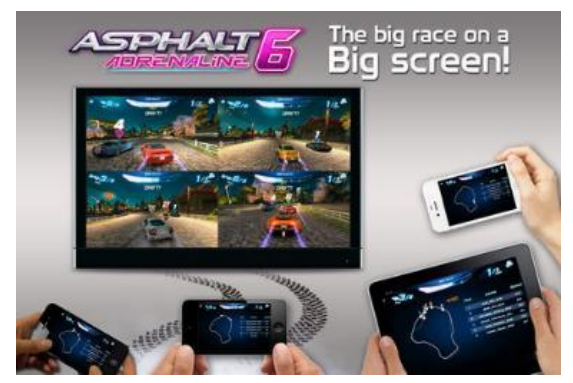

Figure 14. Social interaction (Asphalt Adrenaline [39])

\subsection{Bioinformatics interaction}

Bioinformatics interaction is the interaction that uses unique biodata of the player who interacts with the game. Examples of this interaction are finger scan [40], iris scan, heart rates (show in Figure 15) and eye movements. This interaction type can be used to identify real users, choose profiles, and solve bot problems.

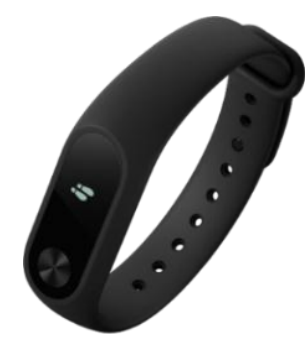

Figure 15. Bioinformatics interaction (Mi Band2 [41]) 
Figure 16 shows the summary of all 14 interactions. Techniques that can be used in mobile game development are also included in each interaction. Interactions on the left side are those found in other related studies, which include 1) Touch interaction 2) Motion/Movement interaction 3) Video interaction 4) Sound interaction and 5) Special purpose interaction. Interactions on the right side are newly presented in this study, which include 1) Location interaction 2) Date/Time interaction 3) Weather interaction 4) Light interaction 5) Proximity interaction 6) Network interaction 7) Social interaction 8) Bioinformatics interaction and 9) Electroencephalography (EEG) interaction. By combining these two groups of interactions together, a complete list of mobile game interactions was constructed, which will be useful for mobile game designers and developers who want to design or develop a mobile game with various interactions. In addition, it will also help reduce the time in designing a mobile game.

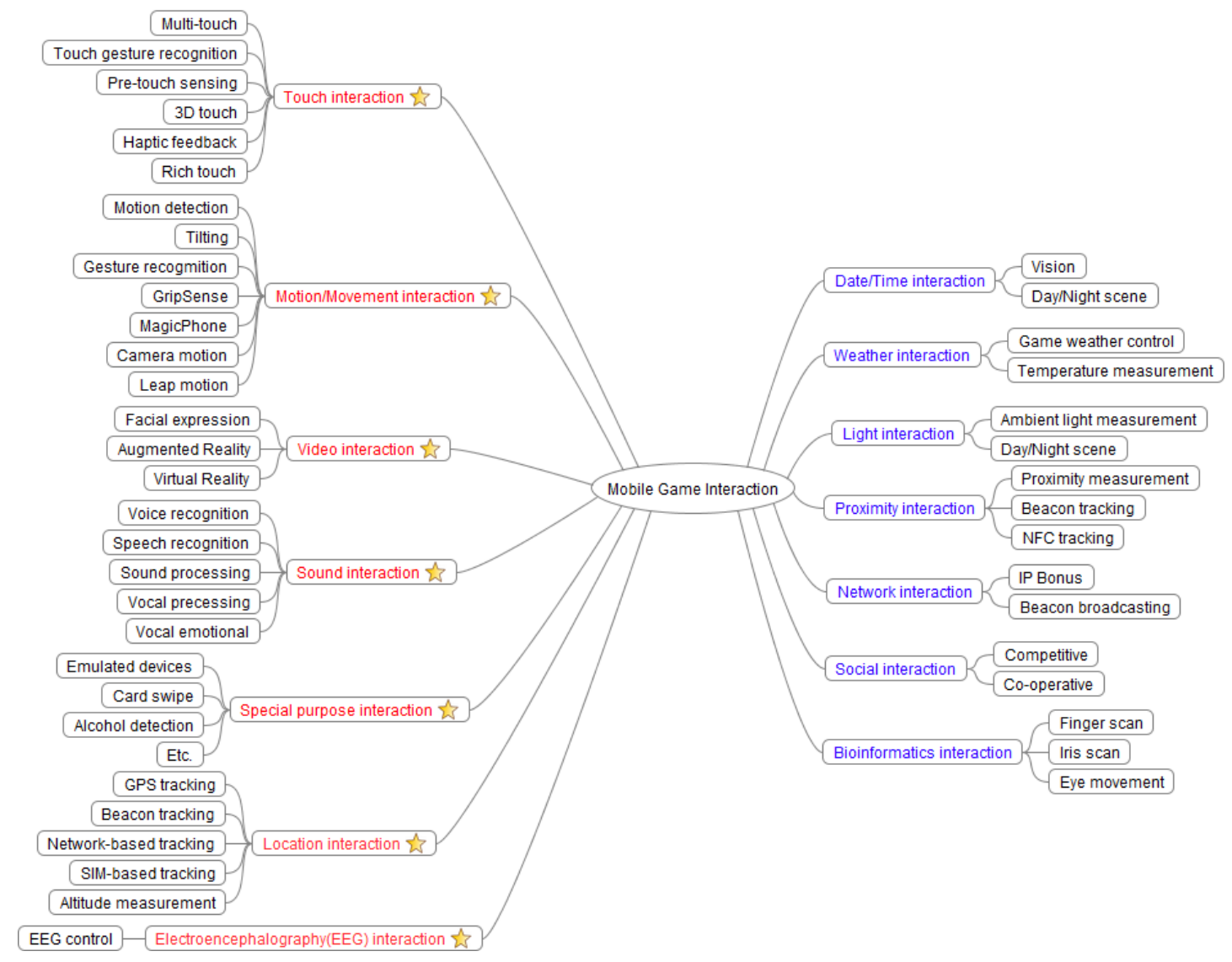

Figure 16. Category of mobile game interactions

\section{GAME INTERACTION EXAMPLES}

\subsection{Successful mobile games}

The Apple Appstore has an annual ranking of popular games. The winning games must be more prominent than any other games. This section explores the interaction techniques of the Game of the Year and Breakout Hit games for 2014, 2015 and 2016, by using the 14 defined interaction techniques presented in this study.

\subsubsection{6: Clash Royale [42]}

Clash Royale is a MOBA (Multiplayer Online Battle Arena) game where players fight with other players in a one-on-one competition with the goal of destroying the opponent's tower. This game consists of two interaction techniques: 1) Touch interaction and 2) Social interaction.

\subsubsection{6: Pokémon GO [28]}

"Pokémon GO" is one of the best games in 2016. This game utilizes AR and location-based techniques in creating new experiences for the players. The game includes a number of interactions: 1) Touch 
interaction 2) Video interaction 3) Location interaction 4) Social interaction 5) Special purpose interaction 6) Weather interaction and 7) Light interaction.

\subsubsection{5: Lara Croft GO [43]}

Square Enix had launched a new game in the Tomb Raider series in 2015. The game was an action puzzle and turn based on smartphones and tablets. Lara Croft GO is a semi-puzzler game. By using isometric view from above, the game focuses on solving puzzles. Only touch interaction is used in the game.

\subsubsection{4: Threes! [44]}

Threes! is a puzzle game on Android and iOS devices. Gameplay is to combine numbers up to three and take the number three to the top of the game. The game lets the player use the finger to move the number up and down or left and right around the screen. This game uses only touch interaction.

The result of the study was that most games use one to two interaction techniques, i.e. touch interaction and social interaction. The interaction of other patterns were rarely seen, but in 2016, Pokémon GO had used seven different interaction techniques. Pokémon GO had earned the Breakout Hit Award, and the game provided an exhilarating experience to the players. From the above mentioned games, it can be seen that adopting the appropriate interaction techniques and using a wide range of them can make the games more interesting and successful.

\subsection{Game interactions for each game genre on mobile devices}

From the 14 types of interactions mentioned above and the interactions from the successful mobile games, this section introduces how to apply these interactions in eight different game genres as shown in Table 3.

Table 3. Interactions for Game Genres on Mobile Devices

\begin{tabular}{lcccccccc}
\hline \multicolumn{1}{c}{ Interactions } & Action & Puzzle & Idle & SIM/Management & Strategy & Sports & Racing & RPG \\
\hline 1. Touch & $\checkmark$ & $\checkmark$ & $\checkmark$ & $\checkmark$ & $\checkmark$ & $\checkmark$ & $\checkmark$ & $\checkmark$ \\
2. Motion/Movement & $\checkmark$ & - & $\checkmark$ & - & - & $\checkmark$ & $\checkmark$ & - \\
3. Video & $\checkmark$ & $\checkmark$ & - & $\checkmark$ & - & $\checkmark$ & $\checkmark$ & - \\
4. Sound & $\checkmark$ & $\checkmark$ & $\checkmark$ & $\checkmark$ & $\checkmark$ & $\checkmark$ & $\checkmark$ & $\checkmark$ \\
5. Special purpose & $\checkmark$ & $\checkmark$ & $\checkmark$ & $\checkmark$ & $\checkmark$ & $\checkmark$ & $\checkmark$ & $\checkmark$ \\
6. Location & - & $\checkmark$ & - & $\checkmark$ & - & - & - & - \\
7. EEG & $\checkmark$ & $\checkmark$ & $\checkmark$ & $\checkmark$ & $\checkmark$ & $\checkmark$ & $\checkmark$ & $\checkmark$ \\
8. Date/Time & - & - & $\checkmark$ & $\checkmark$ & $\checkmark$ & $\checkmark$ & - \\
9. Weather & - & - & $\checkmark$ & $\checkmark$ & $\checkmark$ & $\checkmark$ & - \\
10. Light & - & - & $\checkmark$ & $\checkmark$ & $\checkmark$ & $\checkmark$ & - \\
11. Proximity & $\checkmark$ & $\checkmark$ & $\checkmark$ & $\checkmark$ & $\checkmark$ & $\checkmark$ & $\checkmark$ \\
12. Network & $\checkmark$ & $\checkmark$ & $\checkmark$ & $\checkmark$ & $\checkmark$ & $\checkmark$ & $\checkmark$ & $\checkmark$ \\
13. Social & $\checkmark$ & $\checkmark$ & $\checkmark$ & $\checkmark$ & $\checkmark$ & $\checkmark$ & $\checkmark$ & $\checkmark$ \\
14. Bioinformatics & - & $\checkmark$ & - & $\checkmark$ & - & - & - & - \\
\hline
\end{tabular}

\section{CONCLUSION}

This study had gathered, categorized, and proposed a complete set of mobile game interactions. The result showed that mobile game interactions can be both natural and non-natural. There are many exciting mobile game interactions due to various built-in sensors of the mobile devices. These mobile game interactions enable developers to create innovative games with fresh, new gaming experiences for players.

Mobile game interactions found in past research studies consisted of only seven major interactions including: 1) Touch interaction 2) Motion/Movement interaction 3) Video interaction 4) Sound interaction 5) Special purpose interaction 6) Location interaction and 7) Electroencephalography (EEG) interaction. However, it was found that there were some missing interactions from the literature reviews. This study therefore proposed seven more interactions including: 1) Date/Time interaction 2) Weather interaction 3) Light interaction 4) Proximity interaction 5) Network interaction 6) Social interaction and 7) Bioinformatics interaction.

In conclusion, the complete compilation of 14 interactions for mobile games presented in this manuscript will enable game developers to select appropriate interactions for the success of their mobile games in the market. 


\section{REFERENCES}

[1] N. Mavrogeorgi and Y. Christodoulou, "Complete, Cinematic and Expressive Presentation in Computer Game", in Proceedings of the 1st international conference on PErvasive Technologies Related to Assistive Environments (PETRA '08), Athens, Greece, ACM, pp. 1-4, 2008.

[2] StatCounter, "Desktop vs Mobile vs Tablet Market Share Worldwide", 2016, Retrieved from: http://gs.statcounter.com/platform-market-share/desktop-mobile-tablet, Accessed on January 19, 2017.

[3] K. Curran, "The Future of Web and Mobile Game Development", International Journal of Cloud Computing and Services Science (IJ-CLOSER), vol. 1, no. 1, pp. 25-34, 2012.

[4] I. Hwang, et al., "Toward a Mobile Platform for Pervasive Games", in Proceedings of the first ACM international workshop on Mobile gaming, Helsinki, Finland, ACM, pp. 19-24, 2012.

[5] Steel Media Ltd., "A Brief History of Mobile Games: In the beginning, there was Snake", 2016, Retrieved from: http:/www.pocketgamer.biz/feature/10619/a-brief-history-of-mobile-games-in-the-beginning-there-was-snake, Accessed on January 12, 2017.

[6] K. Browne and C. Anand, "An Empirical Evaluation of user Interfaces for a Mobile Video Game", Entertainment Computing., vol. 3, no. 1, pp. 1-10, 2012.

[7] M. Joselli, et al., "An Architecture for Game Interaction using Mobile”, 2012 IEEE International Games Innovation Conference, Rochester, New York, pp. 1-5, 2012.

[8] J. A. Palacios and D. M. Romano, "Exploring the use of a Respiratory-Computer Interface for Game Interaction", International IEEE Consumer Electronics Society's Games Innovations Conference, London, pp. 154-159, 2009.

[9] S. Cao, et al., "Research on Interaction Design of Chinese Cooking Game Based on Handheld Mobile Devices", 2014 Sixth International Conference on Intelligent Human-Machine Systems and Cybernetics, Hangzhou, pp. 164-167, 2014.

[10] D. Cordeiro, et al., "ARZombie: A Mobile Augmented Reality Game with Multimodal Interaction", 7th International Conference on Intelligent Technologies for Interactive Entertainment (INTETAIN), Turin, pp. 22-31, 2015.

[11] S. Spanogianopoulos, et al., "Human Computer Interaction using Gestures for Mobile Devices and Serious Games: A Review", 2014 International Conference on Interactive Mobile Communication Technologies and Learning (IMCL2014), Thessaloniki, pp. 310-314, 2014.

[12] F. R. Ortega FSA, et al., "Interaction Design for 3D User Interfaces. Boca Raton, Florida," CRC Press, 2016.

[13] M. Savari, et al., "Natural Interaction of Game-based Learning for Elasticity", Malaysian Journal of Computer Science, vol. 29, no. 4, pp. 314-327, 2016.

[14] K. Hinckley, et al., "Pre-Touch Sensing for Mobile Interaction", in Proceedings of the 2016 CHI Conference on Human Factors in Computing Systems(CHI '16), San Jose, California, USA, ACM, pp. 2869-2881, 2016.

[15] T. Niikura and T. Komuro, "3D Touch Panel Interface using an Autostereoscopic Display", in Proceedings of the 2012 ACM international conference on Interactive tabletops and surfaces (ITS '12), Cambridge, Massachusetts, USA, ACM, pp. 295-298, 2012.

[16] A. Badshah, et al., "GyroTab: A Handheld Device that Provides Reactive Torque Feedback", in Proceedings of the SIGCHI Conference on Human Factors in Computing Systems (CHI '12), Austin, Texas, USA, ACM, pp. 3153-3156, 2012.

[17] X. Zhao, et al., "Evaluation of Visuo-haptic Feedback in a 3D Touch Panel Interface", in Proceedings of the Ninth ACM International Conference on Interactive Tabletops and Surfaces (ITS '14), Dresden, Germany, ACM, pp. 299-304, 2014.

[18] UX Magazine, "Improving Mobile Interfaces with Rich-Touch Interactions", 2014, Retrieved from: http://uxmag.com/articles/improving-mobile-interfaces-with-rich-touch-interactions, Accessed on January 15, 2017.

[19] K. Kiili and S. Merilampi, "Developing Engaging Exergames with Simple Motion Detection", in Proceedings of the 14th International Academic MindTrek Conference: Envisioning Future Media Environments (MindTrek '10), Tampere, Finland, ACM, pp. 103-110, 2010.

[20] Y. Du, et al., "Tilt \& Touch: Mobile Phone for 3D Interaction", in Proceedings of the 13th International Conference on Ubiquitous Computing (UbiComp '11), ACM, pp. 485-486, 2011.

[21] K. Murao, et al., "Evaluation Study on Sensor Placement and Gesture Selection for Mobile Devices", in Proceedings of the 11th International Conference on Mobile and Ubiquitous Multimedia (MUM '12), Ulm, Germany, ACM, pp. 1-8, 2012.

[22] J. Kela, et al., "Accelerometer-based Gesture Control for a Design Environment", Personal Ubiquitous Comput., vol. 10, no. 5, pp. 285-299, 2006.

[23] M. Goel, et al., "GripSense: Using Built-in Sensors to Detect hand Posture and Pressure on Commodity Mobile Phones", in Proceedings of the 25th annual ACM symposium on User interface software and technology (UIST '12), Cambridge, Massachusetts, USA, ACM, pp. 545-554, 2012.

[24] J. Wu, et al., "MagicPhone: Pointing \& Interacting", in Proceedings of the 12th ACM International Conference Adjunct Papers on Ubiquitous Computing - Adjunct (UbiComp '10 Adjunct), Copenhagen, Denmark, ACM, pp. 451-452, 2010.

[25] X. Liu, et al., "Fast Camera Motion Estimation for Hand-held Devices and Applications", in Proceedings of the 4th International Conference on Mobile and Ubiquitous Multimedia (MUM '05), Christchurch, New Zealand, ACM, pp. 103-108, 2005. 
[26] Leap Motion, Inc., “Leap Motion,” 2010, Retrieved from: https://www.leapmotion.com, Accessed on January 29 , 2017.

[27] A. H. Basori and H. M. A. AlJahdali, "Emotional Facial Expression based on Action Units and Facial Muscle", International Journal of Electrical and Computer Engineering (IJECE), vol. 6, no. 5, pp. 2478-2487, 2016.

[28] Niantic, Inc., "Pokémon GO", 2016, Retrieved from: https://itunes.apple.com/us/app/pokémon-go/id1094591345, Accessed on February 3, 2017.

[29] S. Moon and K. C. Sim, "Design and Implementation of the Note-taking Style Haptic Voice Recognition for Mobile Devices", in Proceedings of the 14th ACM International Conference on Multimodal Interaction (ICMI '12), Santa Monica, California, USA, ACM, pp. 533-538, 2012.

[30] M. Matton and K. Braeckman, "Speech Recognition Tools in a Media Retrieval System", in Proceedings of the 2011 ACM International Workshop on Automated Media Analysis and Production for Novel TV Services (AIEMPro '11), Scottsdale, Arizona, USA, ACM, pp. 19-24, 2011.

[31] G. Gokul, et al., "Real Time Sound Processing on Android", in Proceedings of the 14th International Workshop on Java Technologies for Real-Time and Embedded Systems (JTRES '16), Lugano, Switzerland, ACM, pp. 1-10, 2016.

[32] Y. Gu, et al., "Vocal Emotion Recognition with Log-Gabor Filters", in Proceedings of the 5th International Workshop on Audio/Visual Emotion Challenge (AVEC '15), Brisbane, Australia, ACM, pp. 25-31, 2015.

[33] Niantic, Inc., "Pokémon GO Plus", 2016, Retrieved from: https://www.pokemongo.com/en-us/pokemon-go-plus, Accessed on January 3, 2018.

[34] Tribe.Red, "Moomin Move", 2017, Retrieved from: https://play.google.com/store/apps/details?id=red.tribe. muumin.world, Accessed on January 3, 2018.

[35] NeuroSky, "EEG Headsets", 2015, Retrieved from: https://store.neurosky.com, Accessed on January 30, 2017

[36] GRAVITY Co., Ltd., "Ro: Idle Poring," 2017, Retrieved from: https://itunes.apple.com/th/app/ro-idleporing/id1272462758, Accessed on January 3, 2018.

[37] Konami Computer Entainment Japan, Boktai, “The Sun Is in Your Hand”, Japan, Konami, 2003.

[38] Apple Inc., "iBeacon", 2012, Retrieved from: https://developer.apple.com/ibeacon/, Accessed on February 3, 2017.

[39] Gameloft, "Asphalt 6: Adrenaline”, 2012, Retrieved from: https:/itunes.apple.com/th/app/asphalt-6adrenaline/id400973408, Accessed on February 3, 2017.

[40] J. Han, "Fingerprint Authentication Schemes for Mobile Devices", International Journal of Electrical and Computer Engineering (IJECE), vol. 5 no. 3, pp. 579-585, 2015.

[41] Xiaomi, "Mi Band2", 2016, Retrieved from: http://www.mi.com/en/miband2/, Accessed on January 3, 2018.

[42] Supercell, "Clash Royale", 2016, Retrieved from: https://itunes.apple.com/th/app/clashroyale/id1053012308?mt=8, Accessed on February 3, 2017.

[43] SQUARE ENIX INC, "Lara Croft GO," 2015, Retrieved from: https:/itunes.apple.com/th/app/lara-croftgo/id971304016, Accessed on February 3, 2017.

[44] Sirvo LLC, “Threes!”, 2014, Retrieved from: https:/itunes.apple.com/us/app/threes/id779157948, Accessed on February 3, 2017.

\section{BIOGRAPHIES OF AUTHORS}

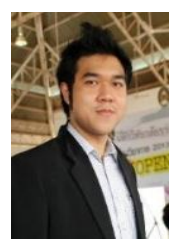

Pitsanu Chaichitwanidchakol received a B.Eng. and M.Eng. degree in computer engineering from Khon Kaen University, Thailand in 2010 and 2012 respectively. He has published many publications in the area of Human Computer Interaction, Video Game Design and Ubiquitous Computing.

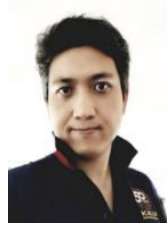

Witcha Feungchan is an assistant professor at the Faculty of Engineering (Computer Engineering), Khon Kaen University where he has been a faculty member since 2002. He completed his Ph.D. in electronic systems engineering at the University of Regina, Canada in 2009. He has made contributions to many publications in the areas of Video Games Design, Serious Games, Ubiquitous Computing, Virtual Reality, and Educational Technology. Witcha has also collaborated actively with multidisciplinary teams in engineering, computer science, and education. 